\title{
A COUPLED KELLER-SEGEL-STOKES MODEL: GLOBAL EXISTENCE FOR SMALL INITIAL DATA AND BLOW-UP DELAY*
}

\author{
ALEXANDER LORZ ${ }^{\dagger}$
}

\begin{abstract}
We study a system consisting of the elliptic-parabolic Keller-Segel equations coupled to Stokes equations by transport and gravitational forcing. We show global-in-time existence of solutions for small initial mass in 2D. In $3 \mathrm{D}$ we establish global existence assuming that the initial $L^{3 / 2}$-norm is small. Moreover, we give numerical evidence that for this extension of the Keller-Segel system in $2 \mathrm{D}$, solutions exist with mass above $8 \pi$, which is the critical mass for the system without fluid.
\end{abstract}

Key words. Keller-Segel, chemotaxis, Stokes, global existence, blow-up.

AMS subject classifications. 35K55, 35Q92, 35Q35, 92C17.

\section{Motivation}

The Keller-Segel system modelling chemotaxis is a very well studied model in mathematical biology. In the following, we investigate a system consisting of the elliptic-parabolic Keller-Segel equations coupled to Stokes equations:

$$
\left\{\begin{array}{l}
u \cdot \nabla c=\Delta c+n-a_{1} c, \\
n_{t}+u \cdot \nabla n=\Delta n-\nabla \cdot(\chi n \nabla c), \\
a_{2} u_{t}+\nabla P-\eta \Delta u+n \nabla \phi=0, \\
\nabla \cdot u=0 .
\end{array}\right.
$$

Here $c$ denotes the concentration of a chemical, $n$ a cell density, and $u$ a fluid velocity field described by Stokes equations. The fluid couples to $n$ and $c$ through transport and gravitational forcing modelled by $\nabla \phi$. The pressure $P$ can be seen as the Lagrange multiplier enforcing the incompressibility constraint. The chemical $c$ diffuses, it is produced by the cells and it degrades. The cell density diffuses and it moves in the direction of the chemical gradient. The constant $a_{1} \geq 0$ measures self-degradation of the chemical and the constants $a_{2} \geq 0, \eta>0$ determine the evolution undergone by $u$.

The motivation for this model comes from experiments described in $[21,30,33]$ for the case of bacteria consuming the chemical. There the authors observed large-scale convection patterns in a water drop sitting on a glass surface containing oxygensensitive bacteria, oxygen diffusing into the drop through the fluid-air interface and they proposed this model:

$$
\left\{\begin{array}{l}
c_{t}+u \cdot \nabla c=\Delta c-n f(c), \\
n_{t}+u \cdot \nabla n=\Delta n-\nabla \cdot(n \chi(c) \nabla c), \\
u_{t}+u \cdot \nabla u+\nabla P-\eta \Delta u+n \nabla \phi=0, \\
\nabla \cdot u=0 .
\end{array}\right.
$$

The idea behind this paper is to take these equations and change consumption of the chemical to production i.e. make it the mathematically more interesting case of

\footnotetext{
${ }^{*}$ Received: March 20, 2011; accepted (in revised version): August 2, 2011. Communicated by Benoit Perthame.

${ }^{\dagger}$ Department of Applied Mathematics and Theoretical Physics, University of Cambridge, Wilberforce Road, Cambridge CB3 0WA, UK (lorz@ann.jussieu.fr).
} 
Keller-Segel chemotaxis. An example in biology could be E. coli which can swim and excrete aspartate [1]. The ultimate aim is to study if and how fluid coupling changes the blow-up behaviour.

Assuming a vanishing fluid velocity field $u$, we recover the elliptic-parabolic Keller-Segel equations; see [31]. The problem of global existence vs. blow-up is very well understood for the elliptic-parabolic Keller-Segel model

$$
\left\{\begin{array}{l}
-\Delta c=n, \\
\partial_{t} n+\nabla \cdot(\chi n \nabla c-\nabla n)=0,
\end{array}\right.
$$

in $\mathbb{R}^{2}$. [5] summarizes the results, i.e. there is a critical mass $M_{c r i t}$ such that if the initial mass is below $M_{\text {crit }}$, then there is global-in-time existence, and if the initial mass above $M_{\text {crit }}$, then the solution blows up in finite time. On a bounded domain $\Omega \subset \mathbb{R}^{2}$ the analysis is more involved because boundary effects play an important role. With zero Dirichlet boundary conditions for $c$ and corresponding no-flux conditions on $n$, the results are the same as in full space; see [3]. For homogeneous Neumann boundary conditions, the Keller-Segel system must be changed to

$$
\left\{\begin{array}{l}
-\Delta c=n-\langle n\rangle, \\
\partial_{t} n+\nabla \cdot(\chi n \nabla c-\nabla n)=0,
\end{array}\right.
$$

because we need the right-hand side of the $c$-equation to have zero average. In this case, there are several threshold values: if $\Omega$ is regular then solutions are global if $\chi M<4 \pi$ and may blow up above this threshold, either on the boundary or inside the domain. For more information about this type of boundary conditions; see [22]. For the parabolic-parabolic Keller-Segel model recent progress has been achieved in [10]. For more references on the general Keller-Segel system, the interested reader can refer to recent work $[4,5,10]$. The Keller-Segel system in higher space dimensions has been investigated in $[13,14]$, and results for the system with nonlinear diffusion to prevent blow-up can be found in $[9,24]$. Other ways to model prevention of overcrowding can be found in $[6,7,20]$. Kinetic models for chemotaxis can be found in [12]. Numerics for the Keller-Segel model have been performed in $[17,29]$.

For the system (1.2) and related systems there is a local existence result [28]. Moreover, in [16] the authors proved global existence for a simplified version of (1.2) with weak potential or small initial c. In [15], global existence of solutions to the system (1.2) with nonlinear diffusion is shown. Furthermore, in [27] and [34], global existence results without smallness assumptions are given. To our knowledge, these are the only results on (1.2). However, attention has recently been focused on coupled kinetic-fluid systems first introduced in [8] which have a similar mathematical flavor; we also refer the reader to $[11,19]$ for studies of the Vlasov-Fokker-Planck equation coupled with the compressible or incompressible Navier-Stokes or Stokes equations, where the main tool used to prove the global existence of weak solutions or hydrodynamic limits is an existing entropy inequality.

For the Navier-Stokes and Stokes equations see $[25,26]$ and references therein for detailed mathematical theory.

The paper is structured as follows. In Section 2 we state the problem in detail and give our results on global existence. In Section 3 we prove the global existence of solutions to (1.1) in 2D for small mass in the following steps: obtaining an entropy, using the regularizing effect, and passing to the limit. In Section 4, we give the main technical differences to address the existence issues in 3D for (1.1). Finally, in Section 
5 we give numerical evidence that solutions to (1.1) exists for initial mass larger than $8 \pi$, but for even larger mass blow-up seems to occur. So the blow-up is delayed.

\section{Preliminaries}

We consider the system (1.1) in full space $\mathbb{R}^{d}, d=2,3$. The system must be supplied with initial data $n(t=0, x)=n_{0}(x)$, for $a_{2}>0$ also $u(t=0, x)=u_{0}(x) . \nabla \phi$ is assumed to be in $L^{\infty}\left(\mathbb{R}^{d}\right)$.

Following [5] we call a triple $(c, n, u)$ a weak solution to (1.1) if the following two conditions hold:

(i) for every $T>0,0 \leq t \leq T, n(t, x) \geq 0, c(t, x) \geq 0, x \in \mathbb{R}^{d}$,

$$
\begin{aligned}
& c \in L^{2}\left(0, T ; H^{2}\left(\mathbb{R}^{d}\right)\right) ; c(\cdot, t) \in H_{0}^{1}\left(\mathbb{R}^{d}\right) \text { for a.e. } t, \\
& n(1+|x|+|\ln n|) \in L^{\infty}\left(0, T ; L^{1}\left(\mathbb{R}^{d}\right)\right), \nabla \sqrt{n} \in L^{2}\left(0, T ; L^{2}\left(\mathbb{R}^{d}\right)\right), \\
& u \in L^{2}\left(0, T ; H^{2}\left(\mathbb{R}^{d}\right)\right), a_{2} u_{t} \in L^{2}\left(0, T ; L^{2}\left(\mathbb{R}^{d}\right)\right) ;
\end{aligned}
$$

(ii) it is further required that for $\psi_{1}, \psi_{2}, \psi_{3} \in C_{0}^{\infty}\left(\mathbb{R}^{d}, \mathbb{R}\right)$ and $\tilde{\psi} \in C_{0}^{\infty}\left(\mathbb{R}^{d}, \mathbb{R}^{d}\right)$ with $\nabla \cdot \tilde{\psi}=0$

$$
\begin{aligned}
&-\int_{\mathbb{R}^{d}} \nabla \psi_{1} \cdot u c d x=\int_{\mathbb{R}^{d}}\left(-\nabla \psi_{1} \cdot \nabla c+\psi_{1} n-a_{1} \psi_{1} c\right) d x, \\
& \frac{d}{d t} \int_{\mathbb{R}^{d}} \psi_{2} n d x=\int_{\mathbb{R}^{d}}\left(\nabla \psi_{2} \cdot u n-\nabla \psi_{2} \cdot \nabla n-\chi \nabla \psi_{2} \cdot n \nabla c\right) d x, \\
& a_{2} \frac{d}{d t} \int_{\mathbb{R}^{d}} \tilde{\psi} u d x+\int_{\mathbb{R}^{d}}(\eta \nabla \tilde{\psi} \cdot \nabla u+\tilde{\psi} \cdot n \nabla \phi) d x=0, \\
& \int_{\mathbb{R}^{d}} \nabla \psi_{3} \cdot u d x=0 .
\end{aligned}
$$

The main idea for showing existence is to first establish a bound on $\int_{\mathbb{R}^{d}} n \ln (n)$ and then use the regularizing effect to achieve $L^{p}$-bounds.

Let us define

$$
\mathcal{E}(t):=\int_{\mathbb{R}^{2}} n(t)|\ln (n(t))| d x+\|u(t)\|_{2}^{2}, \quad \mathcal{D}:=\lambda\|\nabla \sqrt{n}\|_{2}^{2}+\eta\|\nabla u\|_{2}^{2} .
$$

and $L_{\sigma}^{q}\left(\mathbb{R}^{d}\right)$ for $1 \leq q \leq \infty$ as the closure of

$$
\left\{v \in C_{0}^{\infty}\left(\mathbb{R}^{d}\right)^{d} \mid \nabla \cdot v=0\right\}
$$

in $L^{q}\left(\mathbb{R}^{d}\right)$. Let us recall (see [18]) that each vector $f \in L^{q}\left(\mathbb{R}^{d}\right)$ is uniquely decomposed as

$$
f=f_{0}+\nabla Q
$$

with some $f_{0} \in L_{\sigma}^{q}\left(\mathbb{R}^{d}\right), Q \in L_{l o c}^{q}\left(\mathbb{R}^{d}\right), \nabla Q \in L^{q}\left(\mathbb{R}^{d}\right)$ and

$$
\|\nabla Q\|_{q} \leq C\|f\|_{q} \quad \text { and } \quad\|Q\|_{L^{q}\left(B_{0}\right)} \leq C\|Q\|_{q},
$$

where $C$ is independent of $f$ and $B_{0}$ is an open ball in $\mathbb{R}^{d}$. The mapping $f \rightarrow f_{0}$ defines a continuous projection $P_{q}$ from $L^{q}\left(\mathbb{R}^{d}\right)^{d}$ onto $L_{\sigma}^{q}\left(\mathbb{R}^{d}\right)$. Now we can define the Stokes operator $A_{q}:=-P_{q} \Delta$ and also the space

$$
D_{q}^{\alpha, s}:=\left\{v \in L^{q}(\Omega) ;\|v\|_{q}+\left(\int_{0}^{\infty}\left\|t^{1-\alpha} A_{q} e^{-t A_{q}} v\right\|_{q}^{s} \frac{d t}{t}\right)^{1 / s}<\infty\right\} .
$$


More information on the Stokes operator and this definition can be found in [18].

The following result holds for all $a_{1}, a_{2}>0$. For simplicity, let us fix $a_{2}=1$.

Theorem 2.1 (2D). Assume $n_{0} \geq 0, \chi, \eta>0, u_{0} \in D_{3 / 2}^{2 / 3,3}$, and

$$
\int_{\mathbb{R}^{2}} n_{0} \ln \left(n_{0}\right)+n_{0}|x|+n_{0} d x<\infty .
$$

There exists a $M_{\text {exist }}>0$ such that if

$$
\int_{\mathbb{R}^{2}} n_{0} d x<M_{\text {exist }}
$$

then there is a global in time weak solution for (1.1) and we have an entropy inequality

$$
\mathcal{E}(t)+\int_{0}^{t} \mathcal{D} d t^{\prime} \leq C+C t+C \int_{0}^{t} \mathcal{E} d t^{\prime}
$$

where $\mathcal{E}$ and $\mathcal{D}$ are given in (2.8) and $0 \leq t$.

REMARK 2.2 (Justification of the assumption $a_{1}, a_{2}>0$ ). Assuming $a_{1}>0$ gives $\int_{\mathbb{R}^{2}} n d x=a_{1} \int_{\mathbb{R}^{2}} c d x$, and this together with a gradient bound achieved below enables us to control a range of $L^{q}$-norms of $c$ which we could not obtain in full space $\mathbb{R}^{2}$ just from the $L^{2}$-bound on the gradient. The reason for $a_{2}=1$ is similar: for the stationary Stokes system in $\mathbb{R}^{2}$ we only obtain a $L^{2}$-gradient bound, which does not allow us to control any $L^{q}$-norm of $u$ itself.

REMARK 2.3 (Reusing techniques from classical Keller-Segel). We recall that the entropy for the classical Keller-Segel reads as

$$
\mathcal{E}_{K S}:=\int_{\mathbb{R}^{2}} n \ln (n)-\frac{\chi}{2} n c d x .
$$

Already when calculating the time evolution of the second term, we see

$$
\begin{aligned}
\frac{d}{d t} \int_{\mathbb{R}^{2}} \frac{\chi}{2} n c d x & =\chi \int_{\mathbb{R}^{2}} c \partial_{t} n+n \partial_{t} c d x \\
& =\chi \int_{\mathbb{R}^{2}} \nabla c \cdot \nabla n+\frac{\chi}{2} n \nabla c \cdot \nabla n+n \partial_{t} c d x .
\end{aligned}
$$

But there is no way to handle the time derivative on $c$.

At least for a large viscosity $\eta$ and sufficiently large mass, one would expect blowup because in this case $u$ is small and the system is therefore close to the Keller-Segel system. But regarding the additional terms in the computation of the second moment of $n$ :

$$
\begin{aligned}
\frac{d}{d t} \int_{\mathbb{R}^{2}}|x|^{2} n d x & =4 \int_{\mathbb{R}^{2}} n d x+2 \int_{\mathbb{R}^{2}} x \cdot u n d x+2 \int_{\mathbb{R}^{2}} n x \cdot \nabla c d x \\
& =4 M+2 \int_{\mathbb{R}^{2}} x \cdot u n d x+2 \int_{\mathbb{R}^{2}}\left(u \cdot \nabla c-\Delta c+a_{1} c\right) x \cdot \nabla c d x,
\end{aligned}
$$

already for $\int_{\mathbb{R}^{2}} x \cdot$ und $d x$ the regularity that we can obtain for $u$ by using $n$ in $L^{1}$ is not enough to estimate this term. 


\section{Existence in $\mathbb{R}^{2}$}

Here we prove Theorem 2.1: We will establish a-priori estimates. We first establish positivity of $n$ and $c$. In the expression for $\frac{d}{d t} \int n \ln (n)$ the term $\int n u \cdot \nabla c$ occurs. In order to bound it, we use an estimate of $\|\nabla c\|_{2}$. We conclude this section by employing a regularizing effect to pass to the limit.

3.1. Positivity. By some standard argument, e.g. a maximum principle, we can show that for $n_{0} \geq 0$, we have $n(x, t) \geq 0$ for almost all $t$; cf. [2]. The Equation $(1.1)_{2}$ leads to

$$
\frac{d}{d t} \int_{\mathbb{R}^{2}} n d x=0
$$

So the $L^{1}$-norm of $n$, also called its mass, is conserved.

Moreover, also by a maximum principle we obtain $c(x, t) \geq 0$ for almost all $t$. Therefore, the $L^{1}$-norm of $c$ is also conserved:

$$
a_{1}\|c\|_{1}=\|n\|_{1} .
$$

3.2. $\|\nabla c\|_{2}$ bound. Multiplying the Equation (1.1) 1 by $c$ and using the Gagliardo-Nirenberg inequality

$$
\|c\|_{q^{\prime}} \leq C\|\nabla c\|_{2}^{1-1 / q^{\prime}}\|c\|_{1}^{1 / q^{\prime}}
$$

we obtain

$$
\begin{aligned}
& \int_{\mathbb{R}^{2}}(c u \cdot \nabla c) d x+\|\nabla c\|_{2}^{2}=\int_{\mathbb{R}^{2}} n c d x-a_{1}\|c\|_{2}^{2}, \\
& \|\nabla c\|_{2}^{2} \leq\|n\|_{q}\|c\|_{q^{\prime}} \leq C\|n\|_{q}\|\nabla c\|_{2}^{1-1 / q^{\prime}}\|c\|_{1}^{1 / q^{\prime}} \leq C\|n\|_{q}\|\nabla c\|_{2}^{1 / q}\|c\|_{1}^{1-1 / q},
\end{aligned}
$$

with $1 / q+1 / q^{\prime}=1$ and $1<q^{\prime}, q<\infty$. Therefore it follows using the GagliardoNirenberg inequality for $1 \leq q \leq 2$,

$$
\|n\|_{q}=\|\sqrt{n}\|_{2 q}^{2} \leq C\left(\|\nabla \sqrt{n}\|_{2}^{1-1 / q}\|\sqrt{n}\|_{2}^{1 / q}\right)^{2}=C\|\nabla \sqrt{n}\|_{2}^{2-2 / q}\|n\|_{1}^{1 / q},
$$

that

$$
\begin{aligned}
\|\nabla c\|_{2} & \leq C\|n\|_{q}^{q /(2 q-1)}\|c\|_{1}^{(q-1) /(2 q-1)} \leq \frac{C}{a_{1}^{(q-1) /(2 q-1)}}\|n\|_{q}^{q /(2 q-1)}\|n\|_{1}^{(q-1) /(2 q-1)} \\
& \leq \frac{C}{a_{1}^{(q-1) /(2 q-1)}}\left(\|\nabla \sqrt{n}\|_{2}^{2-2 / q}\|n\|_{1}^{1 / q}\right)^{q /(2 q-1)}\|n\|_{1}^{(q-1) /(2 q-1)} \\
& =\frac{C}{a_{1}^{(q-1) /(2 q-1)}}\|\nabla \sqrt{n}\|_{2}^{(2 q-2) /(2 q-1)}\|n\|_{1}^{q /(2 q-1)}
\end{aligned}
$$

3.3. Estimate of the $L^{1}$-norm of $n u \cdot \nabla c$. Let us work on the term $(u \cdot \nabla c) n$ : Using (3.3) with $q=9 / 8$ and the inequality

$$
\|u\|_{\infty} \leq C\|u\|_{2}^{2 / 5}\left\|D^{2} u\right\|_{3 / 2}^{3 / 5}
$$


we arrive at

$$
\int_{\mathbb{R}^{2}}|u \cdot \nabla c| n d x \leq\|u\|_{\infty}\|\nabla c\|_{2}\|n\|_{2} \leq C\|u\|_{2}^{2 / 5}\left\|D^{2} u\right\|_{3 / 2}^{3 / 5}\|\nabla \sqrt{n}\|_{2}^{6 / 5}\|n\|_{1}^{7 / 5} .
$$

Integrating over time and applying Hölder's inequality, we obtain

$$
\begin{aligned}
& \int_{0}^{t} \int_{\mathbb{R}^{2}}|u \cdot \nabla c| n d x d t^{\prime} \\
\leq & C\left(\int_{0}^{t}\|u\|_{2}^{2} d t^{\prime}\right)^{1 / 5}\left(\int_{0}^{t}\left\|D^{2} u\right\|_{3 / 2}^{3} d t^{\prime}\right)^{1 / 5}\left(\int_{0}^{t}\|\nabla \sqrt{n}\|_{2}^{2} d t^{\prime}\right)^{3 / 5}\|n\|_{1}^{7 / 5} .
\end{aligned}
$$

Now we recall a regularity result for the Stokes equations:

TheOREM 3.1 (from [18]). Assume $\Omega=\mathbb{R}^{d}$ or $\Omega \subset \mathbb{R}^{d}$ is a smooth bounded domain. Let $1<s, q<\infty$. Then for every $f \in L^{s}\left((0, T) ; L^{q}(\Omega)\right)$ and $u_{0} \in D_{q}^{1-1 / s, s}$ there exists a unique solution of the Stokes system

$$
\left\{\begin{array}{l}
u_{t}+\nabla P-\eta \Delta u=f \\
\nabla \cdot u=0
\end{array}\right.
$$

satisfying

$$
\int_{0}^{t}\left\|D^{2} u\right\|_{q}^{s} d t^{\prime}+\int_{0}^{t}\left\|\partial_{t} u\right\|_{q}^{s} d t^{\prime} \leq C\left(\int_{0}^{t}\|f\|_{q}^{s} d t^{\prime}+\left\|u_{0}\right\|_{D_{q}^{1-1 / s, s}}^{s}\right) .
$$

Using this, we estimate the second term above (by means of (3.2)) as follows:

$$
\int_{0}^{t}\left\|D^{2} u\right\|_{3 / 2}^{3} d t^{\prime} \leq C\left(\int_{0}^{t}\|n\|_{3 / 2}^{3} d t^{\prime}+\left\|u_{0}\right\|_{D_{3 / 2}^{2 / 3,3}}^{3}\right) \leq C \int_{0}^{t}\|\nabla \sqrt{n}\|_{2}^{2}\|n\|_{1}^{2} d t^{\prime}+C .
$$

Therefore with

$$
a b \leq C(\delta) a^{5}+\delta b^{5 / 4}
$$

we have

$$
\int_{0}^{t} \int_{\mathbb{R}^{2}}|u \cdot \nabla c| n d x d t^{\prime} \leq C(\delta) \int_{0}^{t}\|u\|_{2}^{2} d t^{\prime}+\delta \int_{0}^{t}\|\nabla \sqrt{n}\|_{2}^{2} d t^{\prime}+C\left(n_{0}, u_{0}\right) .
$$

3.4. $n \ln (n)$ estimate. Now we have all the necessary tools to establish the bound on $\int n \ln (n)$. Multiplying the Equation $(1.1)_{2}$ by $\ln (n)$, integrating over $\mathbb{R}^{2}$, integrating by parts, and using Equation $(1.1)_{1}$ gives

$$
\begin{aligned}
& \frac{d}{d t} \int_{\mathbb{R}^{2}} n \ln (n) d x=\int_{\mathbb{R}^{2}} n_{t} \ln (n) d x=\int_{\mathbb{R}^{2}}-\frac{|\nabla n|^{2}}{n}+\chi \nabla n \cdot \nabla c d x \\
&=\int_{\mathbb{R}^{2}}-\frac{|\nabla n|^{2}}{n}-\chi n \Delta c d x=\int_{\mathbb{R}^{2}}-\frac{|\nabla n|^{2}}{n}-\chi n\left(u \cdot \nabla c-n+a_{1} c\right) d x .
\end{aligned}
$$

Using the Gagliardo-Nirenberg inequality

$$
\|n\|_{2}^{2} \leq K\|n\|_{1}\|\nabla \sqrt{n}\|_{2}^{2},
$$


and integrating over $(0, t)$, gives

$$
\begin{aligned}
& \int_{\mathbb{R}^{2}} n(t) \ln (n(t)) d x+\int_{0}^{t}\left(4-\chi K\|n\|_{1}\right)\|\nabla \sqrt{n}\|_{2}^{2} d t^{\prime} \\
\leq & \int_{\mathbb{R}^{2}} n_{0} \ln \left(n_{0}\right) d x-\chi \int_{0}^{t} \int_{\mathbb{R}^{2}}(u \cdot \nabla c) n d x d t^{\prime} .
\end{aligned}
$$

Multiplying the Equation (1.1) 3 with $u$ and integrating over $\mathbb{R}^{2}$ gives

$$
\frac{1}{2} \frac{d}{d t}\|u\|_{2}^{2}+\eta\|\nabla u\|_{2}^{2}=-\int_{\mathbb{R}^{2}} n \nabla \phi \cdot u d x \leq\|\nabla \phi\|_{\infty}\|n\|_{2}\|u\|_{2} \leq \frac{\chi \delta}{2}\|\nabla \sqrt{n}\|_{2}^{2}+C\|u\|_{2}^{2} .
$$

Integrating also this inequality over $(0, t)$, adding it to (3.7), and inserting (3.5), we obtain

$$
\begin{aligned}
& \int_{\mathbb{R}^{2}} n(t) \ln (n(t)) d x+\|u(t)\|_{2}^{2}+\int_{0}^{t}\left(4-\chi K\|n\|_{1}-\chi \delta\right)\|\nabla \sqrt{n}\|_{2}^{2} d t^{\prime}+\eta \int_{0}^{t}\|\nabla u\|_{2}^{2} d t^{\prime} \\
\leq & C \int_{0}^{t}\|u(t)\|_{2}^{2} d t^{\prime}+C\left(n_{0}, u_{0}\right) .
\end{aligned}
$$

3.5. Moment control. Since we work in full space $\Omega=\mathbb{R}^{2}$, we have to bound $\int_{\mathbb{R}^{2}} n(t) \ln (n(t)) d x$ from below. In order to do that, we have to control the behavior of $n$ as $|x| \rightarrow+\infty$ similarly to [16]. To perform this task, we multiply $(1.1)_{2}$ by the smooth function $\xi=\sqrt{1+|x|^{2}}$, integrate and use (3.3) with $q=3 / 2$ :

$$
\begin{aligned}
\frac{d}{d t} \int_{\mathbb{R}^{2}} \xi n d x & =\int_{\mathbb{R}^{2}} n u \cdot \nabla \xi d x+\int_{\mathbb{R}^{2}} n \Delta \xi d x+\chi \int_{\mathbb{R}^{2}} n \nabla c \cdot \nabla \xi d x \\
& \leq\|n\|_{1}\|u\|_{\infty}\|\nabla \xi\|_{\infty}+\|n\|_{1}\|\Delta \xi\|_{\infty}+\|n\|_{2}\|\nabla c\|_{2}\|\nabla \xi\|_{\infty} \\
& \leq C\|u\|_{2}^{2 / 5}\left\|D^{2} u\right\|_{3 / 2}^{3 / 5}+C\|\nabla \sqrt{n}\|_{2}\|n\|_{1}^{1 / 2}\|\nabla \sqrt{n}\|_{2}^{1 / 2}\|n\|_{1}^{3 / 4}+C \\
& \leq \delta^{\prime}\|u\|_{2}\left\|D^{2} u\right\|_{3 / 2}^{3 / 2}+\delta^{\prime}\|\nabla \sqrt{n}\|_{2}^{2}+C\left(\delta^{\prime}\right) .
\end{aligned}
$$

We used that $\|\nabla \xi\|_{\infty}$ and $\|\Delta \xi\|_{\infty}$ are bounded. Integrating the inequality over $(0, t)$ gives

$$
\int_{\mathbb{R}^{2}} \xi n(t) d x \leq \delta^{\prime}\left(\int_{0}^{t}\|u\|_{2}^{2} d t^{\prime}\right)^{1 / 2}\left(\int_{0}^{t}\left\|D^{2} u\right\|_{3 / 2}^{3} d t^{\prime}\right)^{1 / 2}+\delta^{\prime} \int_{0}^{t}\|\nabla \sqrt{n}\|_{2}^{2} d t^{\prime}+C t .
$$

Moreover we have

$$
\begin{aligned}
\int_{\mathbb{R}^{2}} n \ln \left(\frac{1}{n}\right) \mathbb{1}_{n \leq 1} d x & \leq \int_{\mathbb{R}^{2}} n \ln \left(\frac{1}{n}\right) \mathbb{1}_{e^{-\xi} \leq n} d x+\int_{\mathbb{R}^{2}} n \ln \frac{1}{n} \mathbb{1}_{n \leq e^{-\xi}} d x \\
& \leq \int_{\mathbb{R}^{2}} \xi n d x+C \int_{\mathbb{R}^{2}} n^{1 / 2} \mathbb{1}_{n \leq e^{-\xi}} d x \\
& \leq C+\int_{\mathbb{R}^{2}} \xi n d x .
\end{aligned}
$$

Combining (3.11), (3.10), and (3.4), it follows that

$$
-\int_{\mathbb{R}^{2}} n(t) \ln (n(t)) \mathbb{1}_{n \leq 1} d x \leq \frac{\chi \delta}{2} \int_{0}^{t}\|\nabla \sqrt{n}\|_{2}^{2} d t^{\prime}+C \int_{0}^{t}\|u\|_{2}^{2} d t^{\prime}+C t+C .
$$


Since

$$
\int_{\mathbb{R}^{2}} n|\ln (n)| d x=\int_{\mathbb{R}^{2}} n \ln (n) d x-2 \int_{\mathbb{R}^{2}} n \ln (n) \mathbb{1}_{n \leq 1} d x,
$$

we obtain from (3.8) and (3.12) that

$$
\begin{gathered}
\int_{\mathbb{R}^{2}} n(t)|\ln (n(t))| d x+\|u(t)\|_{2}^{2}+\int_{0}^{t}\left(4-\chi K\|n\|_{1}-2 \chi \delta\right)\|\nabla \sqrt{n}\|_{2}^{2} d t^{\prime}+\eta \int_{0}^{t}\|\nabla u\|_{2}^{2} d t^{\prime} \\
\leq C \int_{0}^{t}\|u\|_{2}^{2} d t^{\prime}+C+C t
\end{gathered}
$$

Now let us define

$$
M_{\text {exist }}:=\frac{4}{\chi K} .
$$

Therefore if $\left\|n_{0}\right\|_{1}<M_{\text {exist }}$, we can choose $\delta$ small enough, such that $\lambda:=4-\chi K\|n\|_{1}-2 \chi \delta>0$ and we obtain the entropy inequality (2.12)

$$
\mathcal{E}(t)+\int_{0}^{t} \mathcal{D} d t^{\prime} \leq C\left(n_{0}, u_{0}\right)+C t+C \int_{0}^{t} \mathcal{E} d t^{\prime} .
$$

REMARK 3.2. This is the same mass threshold that can be obtained for the ellipticparabolic Keller-Segel model with the methods from [23].

With the entropy inequality at hand, we have

1. $n|\ln (n)| \in L^{\infty}\left((0, T), L^{1}\left(\mathbb{R}^{2}\right)\right)$,

2. $\nabla \sqrt{n} \in L^{2}\left((0, T) \times \mathbb{R}^{2}\right)$,

3. $n|x| \in L^{\infty}\left((0, T), L^{1}\left(\mathbb{R}^{2}\right)\right)$,

4. $u \in L^{\infty}\left((0, T), L^{2}\left(\mathbb{R}^{2}\right)\right) \cap L^{2}\left((0, T), H^{1}\left(\mathbb{R}^{2}\right)\right)$.

Moreover, we can prove the following lemma:

Lemma 3.3. $\Delta c \in L^{2}\left((0, T) \times \mathbb{R}^{2}\right)$.

Proof. From the Equation $(1.1)_{1}$, we arrive at an estimate for $\Delta c$ :

$$
\begin{aligned}
\int_{0}^{T}\|\Delta c(t)\|_{2}^{2} d t & \leq \int_{0}^{T}\left(\|u\|_{\infty}\|\nabla c\|_{2}+\|n\|_{2}+a_{1}\|c\|_{2}\right)^{2} d t \\
& \leq \int_{0}^{T} C\|u\|_{2}^{4 / 5}\left\|D^{2} u\right\|_{3 / 2}^{6 / 5}\|\nabla c\|_{2}^{2}+C\|\nabla \sqrt{n}\|_{2}^{2}\|n\|_{1}+C\|c\|_{1}\|\nabla c\|_{2} d t .
\end{aligned}
$$

Working on the first term, we have

$$
\int_{0}^{T}\|u\|_{2}^{4 / 5}\left\|D^{2} u\right\|_{3 / 2}^{6 / 5}\|\nabla c\|_{2}^{2} d t \leq\|u\|_{L_{t}^{\infty} L_{x}^{2}}^{4 / 5}\left(\int_{0}^{T}\left\|D^{2} u\right\|_{3 / 2}^{3} d t\right)^{2 / 5}\left(\int_{0}^{T}\|\nabla c\|_{2}^{10 / 3} d t\right)^{3 / 5} .
$$

As in (3.8), and using (3.3) with $q=7 / 4$, it follows that

$$
\begin{aligned}
& \int_{0}^{T}\|u\|_{2}^{4 / 5}\left\|D^{2} u\right\|_{3 / 2}^{6 / 5}\|\nabla c\|_{2}^{2} d t \\
\leq & C\|u\|_{L_{t}^{\infty} L_{x}^{2}}^{4 / 5}\left(\int_{0}^{T}\|\nabla \sqrt{n}\|_{2}^{2} d t+C\left(u_{0}\right)\right)^{2 / 5}\left(\int_{0}^{T}\|\nabla \sqrt{n}\|_{2}^{2} d t\right)^{3 / 5} .
\end{aligned}
$$




\subsection{Regularizing effect.}

TheOREM 3.4. Let $t>0$ and $1<p<\infty$. With the hypothesis of Theorem 2.1, there exists a constant $C(t)$ not depending on $\left\|n_{0}\right\|_{p}$ such that

$$
\int_{\mathbb{R}^{2}} n^{p} d x \leq C(t)\left(1+\left(t^{\prime}\right)^{1-p}\right), \quad \forall 0<t^{\prime} \leq t,
$$

i.e. the cell density $n\left(\cdot, t^{\prime}\right)$ belongs to $L^{p}$ for any positive time $t^{\prime}$. The proof works in the same way as in [10] and it uses the bound established in Lemma 3.3. For completeness, we give a sketch of the proof in the appendix.

3.7. Passing to the limit. We approximate the system by

$$
\left\{\begin{array}{l}
u^{\epsilon} \cdot \nabla c^{\epsilon}=\Delta c^{\epsilon}+n^{\epsilon} * \rho^{\epsilon}-c^{\epsilon} \\
n_{t}^{\epsilon}+u^{\epsilon} \cdot \nabla n^{\epsilon}=\Delta n^{\epsilon}-\nabla \cdot\left(\chi n^{\epsilon} \nabla c^{\epsilon}\right), \\
u_{t}^{\epsilon}+\nabla P^{\epsilon}-\eta \Delta u^{\epsilon}+\left(n^{\epsilon} \nabla \phi\right) * \rho^{\epsilon}=0 \\
\nabla \cdot u^{\epsilon}=0
\end{array}\right.
$$

with a standard mollifier $\rho^{\epsilon}$ and mollified versions of $n_{0}, u_{0}$ as initial data $n_{0}^{\epsilon}, u_{0}^{\epsilon}$. All a-priori estimates still hold; e.g. Equation (3.6) becomes

$$
\frac{d}{d t} \int_{\mathbb{R}^{2}} n^{\epsilon} \ln \left(n^{\epsilon}\right) d x=\int_{\mathbb{R}^{2}}-\frac{\left|\nabla n^{\epsilon}\right|^{2}}{n^{\epsilon}}-\chi n\left(u^{\epsilon} \cdot \nabla c^{\epsilon}-n^{\epsilon} * \rho^{\epsilon}+c^{\epsilon}\right) d x .
$$

Here we can estimate

$$
\int_{\mathbb{R}^{2}} n^{\epsilon}\left(n^{\epsilon} * \rho^{\epsilon}\right) d x \leq\left\|n^{\epsilon}\right\|_{2}\left\|n^{\epsilon}\right\|_{2}\left\|\rho^{\epsilon}\right\|_{1}=\left\|n^{\epsilon}\right\|_{2}^{2}
$$

In (3.1), we can estimate instead

$$
\left\|\nabla c^{\epsilon}\right\|_{2}^{2} \leq\left\|n^{\epsilon} * \rho^{\epsilon}\right\|_{q}\left\|c^{\epsilon}\right\|_{q^{\prime}} \leq\left\|n^{\epsilon}\right\|_{q}\left\|\rho^{\epsilon}\right\|_{1}\left\|c^{\epsilon}\right\|_{q^{\prime}} .
$$

Similarly, the estimate for regularity of $u$ still holds. To be able to pass to the limit, we show sufficient compactness.

We proceed similarly to [4].

Bound on $\left\|n^{\epsilon}\right\|_{2}$ : $\quad$ For every $p<\infty$, we have $n^{\epsilon} \in L^{\infty}\left((\delta, T), L^{p}\left(\mathbb{R}^{2}\right)\right)$ for any $\delta \in(0, T)$ from Theorem 3.4.

Bound on $\left\|\nabla n^{\epsilon}\right\|_{2}$ : We have

$$
\left\|n^{\epsilon} \nabla c^{\epsilon}\right\|_{2} \leq\left\|n^{\epsilon}\right\|_{3}\left\|\nabla c^{\epsilon}\right\|_{6} \leq C\left\|n^{\epsilon}\right\|_{3}\left\|\Delta c^{\epsilon}\right\|_{3 / 2},
$$

and working on the second term we obtain

$$
\left\|\Delta c^{\epsilon}\right\|_{3 / 2} \leq\left\|u^{\epsilon}\right\|_{6}\left\|\nabla c^{\epsilon}\right\|_{2}+\left\|n^{\epsilon}\right\|_{3 / 2}+\left\|c^{\epsilon}\right\|_{3 / 2} .
$$

Therefore $n^{\epsilon} \nabla c^{\epsilon}$ is bounded in $L^{\infty}\left((\delta, T), L^{2}\left(\mathbb{R}^{2}\right)\right)$. Now

$$
\frac{d}{d t} \int_{\mathbb{R}^{2}}\left|n^{\epsilon}\right|^{2} d x=-2 \int_{\mathbb{R}^{2}}\left|\nabla n^{\epsilon}\right|^{2} d x+2 \chi \int_{\mathbb{R}^{2}} n^{\epsilon} \nabla n^{\epsilon} \cdot \nabla c^{\epsilon} d x
$$

shows that $\beta:=\left\|\nabla n^{\epsilon}\right\|_{L^{2}\left((\delta, T) \times \mathbb{R}^{2}\right)}$ satisfies the estimate

$$
2 \beta^{2}-2 \chi\left\|n^{\epsilon} \nabla c^{\epsilon}\right\|_{L^{\infty}\left((\delta, T), L^{2}\left(\mathbb{R}^{2}\right)\right)} \beta \leq 2\left\|n^{\epsilon}\right\|_{L^{\infty}\left((\delta, T), L^{2}\left(\mathbb{R}^{2}\right)\right)} .
$$


This implies that $\nabla n$ is bounded in $L^{2}\left((\delta, T) \times \mathbb{R}^{2}\right)$. We define $V:=\left\{v \in H^{1}\left(\mathbb{R}^{2}\right):|x|^{1 / 4} v \in L^{2}\left(\mathbb{R}^{2}\right)\right\}$. Since

$$
\int_{|x|>R} v^{2} \leq R^{-1 / 2} \int_{\mathbb{R}^{2}} \sqrt{|x|} v^{2}
$$

$\mathrm{V}$ embeds compactly in $L^{2}\left(\mathbb{R}^{2}\right)$. Moreover, the estimate

$$
\int_{\mathbb{R}^{2}} \sqrt{|x|}\left(n^{\epsilon}\right)^{2} \leq\left(\int_{\mathbb{R}^{2}}|x| n^{\epsilon}\right)^{1 / 2}\left(\int_{\mathbb{R}^{2}}\left(n^{\epsilon}\right)^{3}\right)^{1 / 2}
$$

shows that $n^{\epsilon}$ is bounded in $L^{2}((\delta, T), V)$. Therefore, the Aubin-Lions lemma gives a strongly convergent subsequence $n^{\epsilon} \rightarrow n$ in $L_{l o c}^{2}\left((\delta, T) \times \mathbb{R}^{2}\right)$. Also from Theorem 3.4 , we obtain enough regularity to make all terms in the definition of weak solutions well-defined.

\section{Existence in $\mathbb{R}^{3}$}

$$
\tilde{\mathcal{E}}(t):=\int_{\mathbb{R}^{3}} n^{3 / 2} d x, \quad \tilde{\mathcal{D}}(t):=\lambda^{\prime} \int_{\mathbb{R}^{3}}\left|\nabla n^{3 / 4}\right|^{2} d x .
$$

Theorem 4.1 (3D). Assume $a_{2}=0, \Omega=\mathbb{R}^{3}, n_{0} \geq 0, \chi, \eta>0$, and

$$
\int_{\mathbb{R}^{3}} n_{0}^{3 / 2}+n_{0}|x|+n_{0} d x<\infty
$$

There exists a $M_{\text {exist }}^{\prime}\left(\left\|n_{0}\right\|_{1}\right)>0$ such that if

$$
\left\|n_{0}\right\|_{3 / 2}<M_{\text {exist }}^{\prime},
$$

then there is a global in time weak solution for (1.1) and we have an entropy inequality

$$
\frac{d}{d t} \tilde{\mathcal{E}}(t)+\tilde{\mathcal{D}}(t) \leq 0
$$

where $\tilde{\mathcal{E}}$ and $\tilde{\mathcal{D}}$ are given in (4.1).

In $3 \mathrm{D}$, the critical norm for the Keller-Segel equations is $L^{3 / 2}$; see [14]. Let us apply the method from before and understand why it does not work in 3D: If we just consider the case without the fluid we have

$$
\frac{d}{d t} \int_{\mathbb{R}^{3}} n^{3 / 2} d x \leq\left[\frac{1}{2} K_{4} \chi\|n\|_{3 / 2}-\frac{4}{3}\right] \int_{\mathbb{R}^{3}}\left|\nabla n^{3 / 4}\right|^{2} d x .
$$

Details can be found in the calculations below. So for $\|n\|_{3 / 2}$ small enough initially, we have $\|n(t)\|_{3 / 2} \leq\left\|n_{0}\right\|_{3 / 2}$. With the non-stationary Stokes system, there is a additive constant on the right-hand side that basically comes from the initial data of the fluid (see the calculations in the $2 \mathrm{D}$-case). So there can be linear growth of $\|n\|_{3 / 2}$, which will eventually destroy the smallness required.

Therefore we restrict ourselves to system (1.1) with $a_{2}=0$. 
Proof of Theorem 4.1. Proof. We first establish an entropy. Multiplying the Equation $(1.1)_{2}$ by $n^{1 / 2}$, integrating over $\mathbb{R}^{3}$, and using the Equation $(1.1)_{1}$ gives

$$
\begin{aligned}
\frac{d}{d t} \int_{\mathbb{R}^{3}} n^{3 / 2} d x & =\int_{\mathbb{R}^{3}}-\frac{4}{3}\left|\nabla n^{3 / 4}\right|^{2}+\frac{1}{2} \chi \nabla n^{3 / 2} \cdot \nabla c d x \\
& =\int_{\mathbb{R}^{3}}-\frac{4}{3}\left|\nabla n^{3 / 4}\right|^{2}-\frac{1}{2} \chi n^{3 / 2} \Delta c d x \\
& =\int_{\mathbb{R}^{3}}-\frac{4}{3}\left|\nabla n^{3 / 4}\right|^{2} d x-\frac{1}{2} \chi n^{3 / 2}\left(u \cdot \nabla c-n+a_{1} c\right) d x .
\end{aligned}
$$

Now working on the equation for $c$ to obtain an estimate for $\|\nabla c\|_{2}$ :

$$
\begin{aligned}
& \int_{\mathbb{R}^{3}}(c u \cdot \nabla c) d x+\|\nabla c\|_{2}^{2}=\int_{\mathbb{R}^{3}} n c d x-a_{1}\|c\|_{2}^{2}, \\
& \|\nabla c\|_{2}^{2} \leq\|n\|_{6 / 5}\|c\|_{6} .
\end{aligned}
$$

Moreover, it follows with $\|v\|_{6} \leq K_{1}\|\nabla v\|_{2}$ that

$$
\|\nabla c\|_{2} \leq K_{1}\|n\|_{6 / 5}
$$

The term $n^{3 / 2}(u \cdot \nabla c)$ can be estimated using $\|v\|_{6} \leq K_{1}\|\nabla v\|_{2}$ and $\|\nabla u\|_{2} \leq K_{2}\left\|D^{2} u\right\|_{6 / 5}$ :

$$
\left\|n^{3 / 2}(u \cdot \nabla c)\right\|_{1} \leq\left\|n^{3 / 2}\right\|_{3}\|u\|_{6}\|\nabla c\|_{2} \leq K_{1}^{2}\left\|\nabla n^{3 / 4}\right\|_{2}^{2} K_{1} K_{2}\left\|D^{2} u\right\|_{6 / 5} K_{1}\|n\|_{6 / 5} .
$$

Now applying the regularity estimates for the stationary Stokes equation $\left\|D^{2} u\right\|_{6 / 5} \leq K_{3}\|n\|_{6 / 5}($ see $[25,26])$ and the Hölder inequality $\|n\|_{6 / 5}^{2} \leq\|n\|_{1}\|n\|_{3 / 2}$, it follows

$$
\left\|n^{3 / 2}(u \cdot \nabla c)\right\|_{1} \leq K_{1}^{4} K_{2} K_{3}\left\|\nabla n^{3 / 4}\right\|_{2}^{2}\|n\|_{6 / 5}^{2} \leq K_{1}^{4} K_{2} K_{3}\left\|\nabla n^{3 / 4}\right\|_{2}^{2}\|n\|_{1}\|n\|_{3 / 2} .
$$

Using

$$
\int_{\mathbb{R}^{3}} n^{5 / 2} d x \leq K_{4} \int_{\mathbb{R}^{3}}\left|\nabla n^{3 / 4}\right|^{2} d x\|n\|_{3 / 2}
$$

we obtain

$$
\begin{aligned}
\frac{d}{d t} \int_{\mathbb{R}^{3}} n^{3 / 2} d x & =\int_{\mathbb{R}^{3}}-\frac{4}{3}\left|\nabla n^{3 / 4}\right|^{2}-\frac{1}{2} \chi n^{3 / 2}\left(u \cdot \nabla c-n+a_{1} c\right) d x \\
& \leq\left[\frac{1}{2}\left(K_{4}+K_{1}^{4} K_{2} K_{3}\|n\|_{1}\right) \chi\|n\|_{3 / 2}-\frac{4}{3}\right] \int_{\mathbb{R}^{3}}\left|\nabla n^{3 / 4}\right|^{2} d x
\end{aligned}
$$

Now let us define

$$
M_{\text {exist }}^{\prime}\left(\|n\|_{1}\right):=\frac{8}{3 \chi\left(K_{4}+K_{1}^{4} K_{2} K_{3}\|n\|_{1}\right)} .
$$

Therefore if $\left\|n_{0}\right\|_{3 / 2}<M_{\text {exist }}^{\prime}$, we set $\lambda^{\prime}:=\frac{4}{3}-\frac{1}{2}\left(K_{4}+K_{1}^{4} K_{2} K_{3}\|n\|_{1}\right) \chi\|n\|_{3 / 2}>0$ and we obtain the entropy inequality (4.4)

$$
\frac{d}{d t} \tilde{\mathcal{E}}(t)+\tilde{\mathcal{D}}(t) \leq 0
$$

With the entropy inequality at hand, we obtain the following:

$n$ is bounded in $L^{5 / 2}\left((0, T) \times \mathbb{R}^{3}\right)$ due to (4.8). Therefore, $u$ is bounded in $L^{5 / 2}\left((0, T) ; W^{2,5 / 2}\left(\mathbb{R}^{3}\right)\right)$ and $c$ is bounded in $L^{5 / 2}\left((0, T) ; W^{2,5 / 2}\left(\mathbb{R}^{3}\right)\right)$. As in the $2 \mathrm{D}$ case, we can use a regularizing effect (for details see [14]) and pass to the limit. 


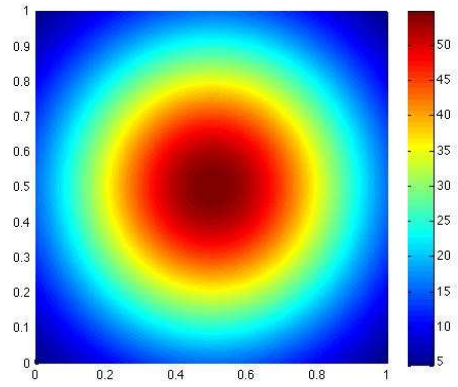

(a) $t=0.01$

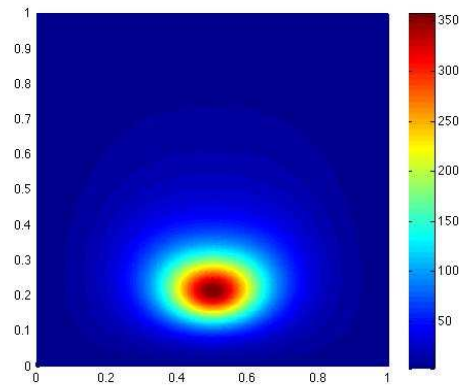

(c) $t=0.2$

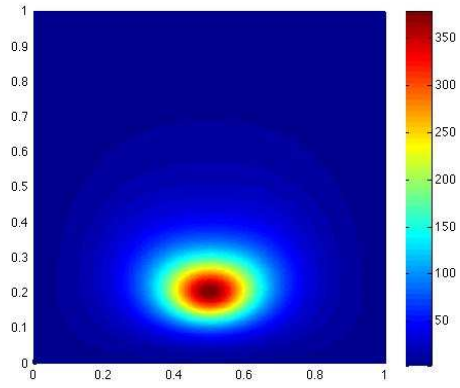

(e) $t=0.75$

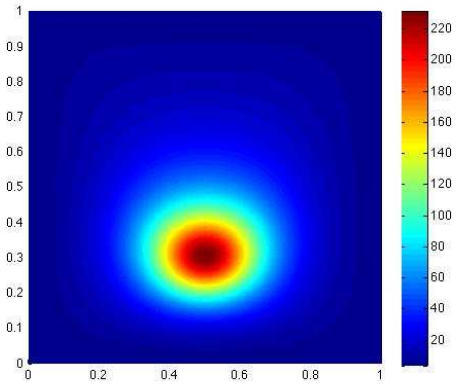

(b) $t=0.1$

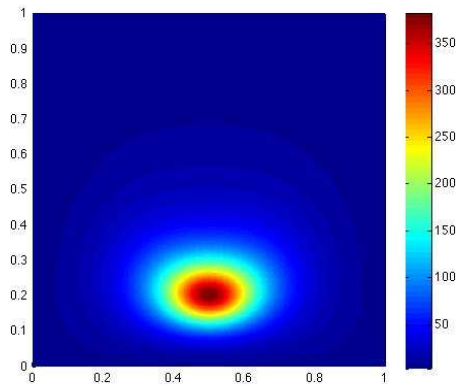

(d) $t=0.5$

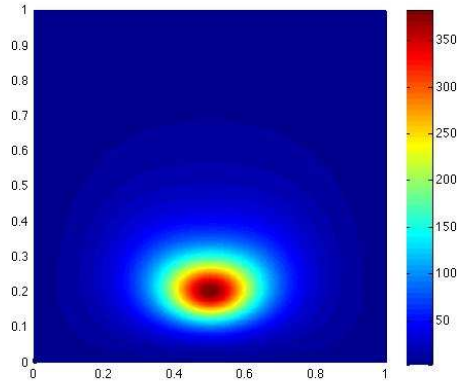

(f) $t=1$

Fig. 5.1: Evolution of the density $n$ with symmetric initial data and mass $M=27$. Although the mass is larger than $8 \pi$, so without the fluid we expect blow-up, with the fluid we see convergence to a steady state.

\section{Numerics}

Finally we would like to illustrate the behavior of the Keller-Segel-fluid system with different numerical examples. In particular, we give evidence that above the critical mass of $8 \pi$ solutions still exist.

All computations have been implemented using the software package FreeFem++ 
[35]. We would like to solve the following system:

$$
\left\{\begin{array}{l}
u \cdot \nabla c=\Delta c+n, \\
n_{t}+u \cdot \nabla n=\Delta n-\nabla \cdot(\chi n \nabla c), \\
\nabla P-\eta \Delta u+n \nabla \phi=0, \\
\nabla \cdot u=0,
\end{array}\right.
$$

with zero Dirichlet boundary conditions for $c$ and $u$ and corresponding masspreserving Neumann conditions for $n$. This system has the advantage that without the fluid there is a mass threshold to separate global existence and finite-time blowup. This is quite different from (1.4), where e.g. all constant states are steady, i.e. for constant initial $n_{0}$ there is always global existence independent of its mass. Moreover, for (5.1) the existence result for small mass holds as for (1.1).

We solve system (5.1) in an iterative manner:

1. Solve the Stokes Equations $(5.1)_{3}$ and $(5.1)_{4}$ with a penalty method; cf. [32] and the Solver Crout. We use a classical Taylor-Hood element technic, i.e. the velocity $u$ is approximated by $P_{2}$ finite elements, and the pressure $P$ is approximated by $P_{1}$ finite elements.

2. Approximate the chemoattractant $c$ by $P_{2}$ finite elements and solve Equation (5.1) 1 with UMFPACK.

3. Perform an implicit Euler finite difference approximation in time for Equation $(5.1)_{2}$, approximate the cell density $n$ by $P_{2}$ finite elements and solve Equation $(5.1)_{1}$ with UMFPACK.

We start with a Gaussian as initial distribution

$$
n_{0}(x, y)=C_{m a s s} \exp \left[-5\left(x-x_{0}\right)^{2}-5\left(y-y_{0}\right)^{2}\right] .
$$

The constant $C_{\text {mass }}$ is chosen initially such that a desired mass in the computational domain is obtained and we set $\chi=1, \eta=1, \nabla \phi=(0 ; 10)$. The test geometry is a square $[0,1] \times[0,1]$ with a mesh consisting of 2500 squares. In the first two examples, we use the mass $M=27>8.5 \pi>8 \pi+1.5$ in the computational domain, i.e. above the critical mass.

In the first example, we set $d t=0.01$ and choose a Gaussian with $x_{0}=y_{0}=0.5$ as initial datum. We observe that the solution does not blow up, although the mass is above the critical value of $8 \pi$; cf. Figure 5.1. Moreover, we see that the solution converges to a steady state. The density maximum moves downwards because of gravity but stops since $c$ vanishes at the boundary. The distribution of the chemical $c$ is shown in Figure 5.2.

Now we investigate various quantities for $t=1$, because this seems to be fairly close to the steady state: Figure 5.3a shows the velocity of the fluid. So the fluid is constantly transporting $n$ and $c$. This is illustrated in Figures 5.3b - 5.3d. Figure 5.3b represents the flux resulting from the chemotaxis / diffusion terms $-(\nabla n-\chi n \nabla c)$, whereas Figure 5.3c shows the flux coming from the fluid contribution un. The total flux $-(\nabla n-\chi n \nabla c-u n)$ is given in Figure 5.3d. It should be noticed that the fluid counteracts the chemotactic flux especially in the high concentration region. To highlight the effect of the fluid, the 0-level set of the scalar product of chemotactic flux and the total flux is plotted, i.e. inside this region the fluid changes the chemotactic flux vector by more than $\pm 90^{\circ}$. 


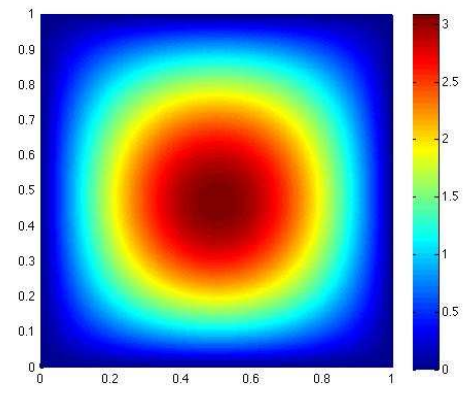

(a) $t=0.01$

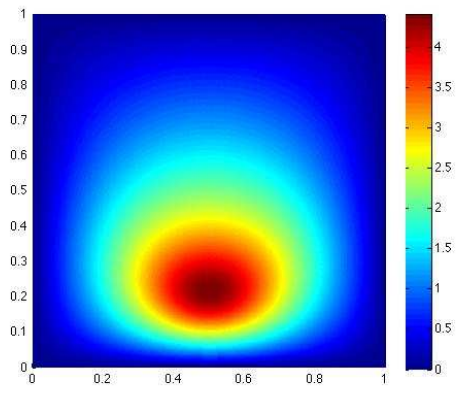

(b) $t=1$

Fig. 5.2: Evolution of the concentration $c$ corresponding to Figure 5.1. Notice, the order of magnitude of $c$ stays the same.

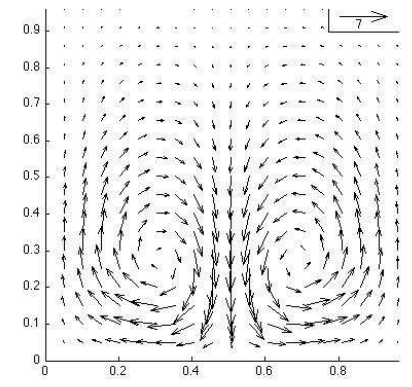

(a) fluid velocity

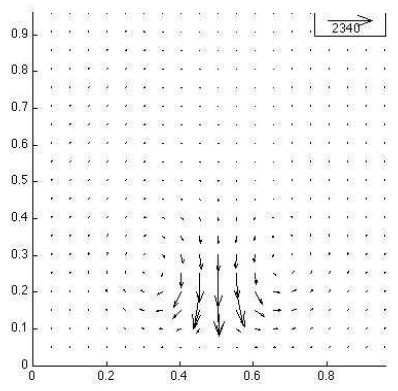

(c) fluid flux

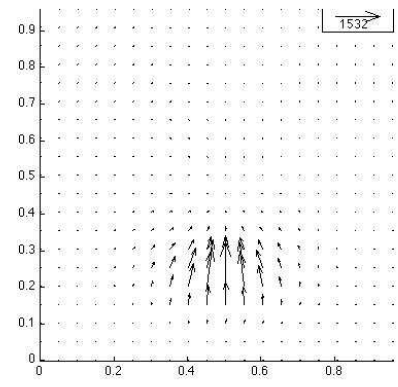

(b) chemotactic flux

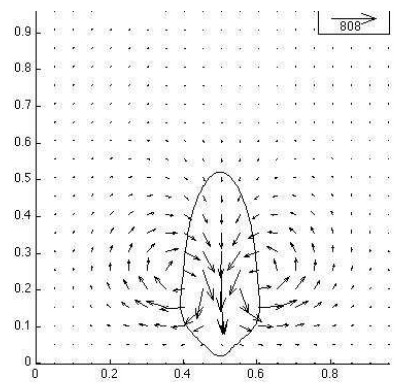

(d) total flux

Fig. 5.3: Fluid velocity field, chemotactic flux $-(\nabla n-\chi n \nabla c)$, fluid flux un and total flux $-(\nabla n-\chi n \nabla c-u n)$ at the steady state. To highlight the effect of the fluid, the 0-level set of the scalar product of chemotactic flux and the total flux is plotted in Figure 5.3d, i.e. inside this region the fluid changes the chemotactic flux vector by more than $\pm 90^{\circ}$. So it can be seen that the fluid has the strongest effect in the high concentration region of $n$. 


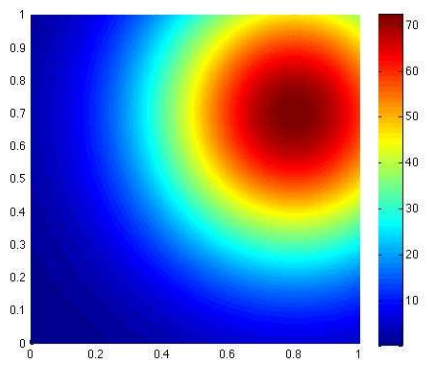

(a) $t=0.01$

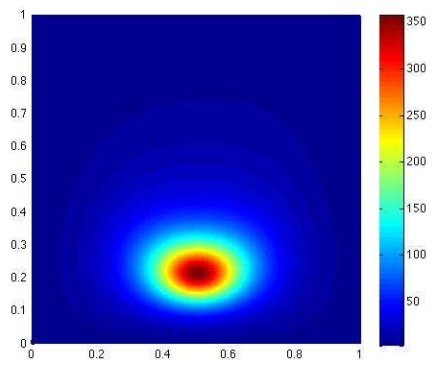

(c) $t=0.2$

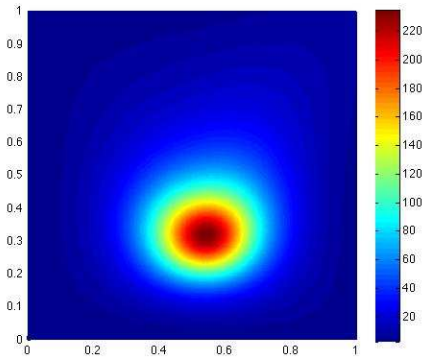

(b) $t=0.1$

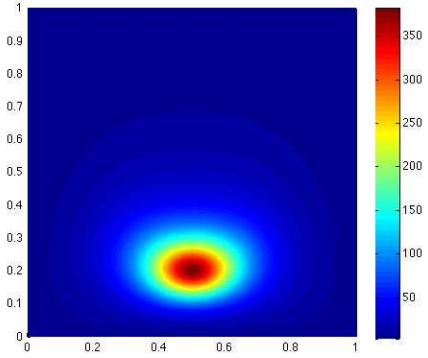

(d) $t=0.5$

Fig. 5.4: Evolution of the density $n$ with non-symmetric initial data and mass $M=27$. For the same mass but different initial data we observe again blow-up prevention and convergence to the same steady state.

Moreover, different initial configurations with the same mass seem to converge to the same steady state; cf. Figure 5.4. This is illustrated by the second example with $d t=0.01$ and $x_{0}=0.8 ; y_{0}=0.7$.

These two results above are stable under mesh and time step refinement. Moreover, if we define

$$
M_{\Delta}:=\left|M-\int_{\Omega} n(t)\right|,
$$

and take its maximum over the first 100 time step, which is less than $10^{-9}$.

In a final step, for even larger mass $M=40$, we observe the development of very high concentration, which might indicate blow-up; see Figure 5.5. Here we set $d t=$ 0.005 and $x_{0}=y_{0}=0.5$.

These first numerical results illustrate the interesting behavior of the Keller-SegelFluid system and can be regarded as a starting point for further research. In particular we would like to use numerical schemes, which are able to couple the Stokes equations with the chemotactial system and capture blow-up e.g. [29] or [17] (for schemes designed to resolve the blow-up for the Keller-Segel system). 


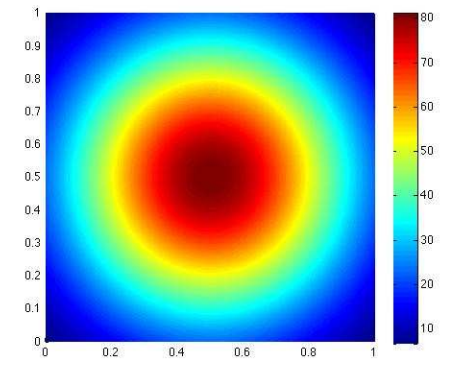

(a) $t=0.005$

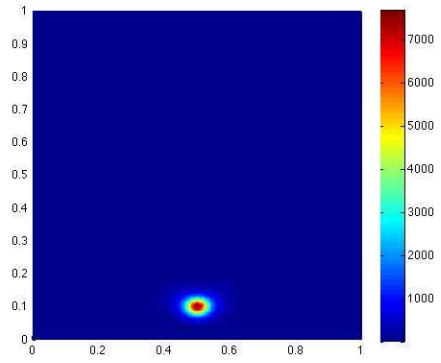

(c) $t=0.11$

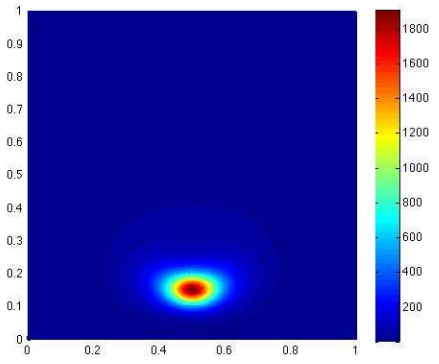

(b) $t=0.075$

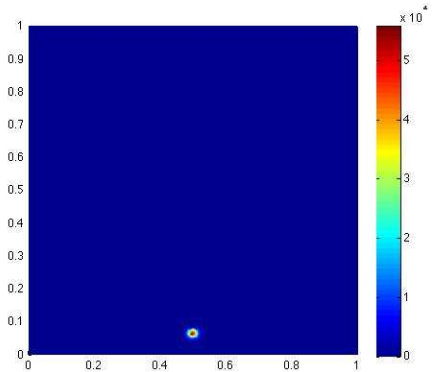

(d) $t=0.145$

Fig. 5.5: Evolution of the density $n$ with symmetric initial data and mass $M=40$. So for higher mass, we observe the formation of very high concentration that might indicate blow-up. Notice that the pictures are taken at much smaller times compared the plots shown before.

Appendix A. The proof for the regularizing effect is taken from [10]:

Proof of Theorem 3.4: $\quad$ Since $\int_{\mathbb{R}^{2}} n \ln (n)(t) d x \leq C(1+t)$ we have

$$
\begin{aligned}
\int_{\mathbb{R}^{2}}(n(x, t)-k)_{d} x+ & \leq \frac{1}{\ln (k)} \int_{\mathbb{R}^{2}}(n(x, t)-k)_{+} \ln (n(x, t)) d x \\
& \leq \frac{1}{\ln (k)} \int_{\mathbb{R}^{2}} n(x, t)(\ln (n(x, t)))_{+} d x
\end{aligned}
$$

This means that there exists a modulus of equi-integrability $\omega(T, k), T>0$, and $k>0$ such that

$$
\int_{\mathbb{R}^{2}}(n(x, t)-k)_{+} \leq \omega(T ; k) \text { and } \lim _{k \rightarrow \infty} \omega(T ; k)=0 .
$$

In this section we follow the by now classical idea to obtain $L^{p}$ bounds for $n$ by using the equi-integrability property (A.1). 
First step: Multiplying the Equation $(1.1)_{2}$ by $(n-k)_{+}^{p-1}$ and integrating over $\mathbb{R}^{2}$ gives

$$
\begin{aligned}
& \frac{d}{d t} \int_{\mathbb{R}^{2}}(n-k)_{+}^{p} d x \\
= & -4 \frac{p-1}{p} \int_{\mathbb{R}^{2}}\left|\nabla(n-k)_{+}^{p / 2}\right|^{2} d x-(p-1) \int_{\mathbb{R}^{2}}(n-k)_{+}^{p} \Delta c d x-p k \int_{\mathbb{R}^{2}}(n-k)_{+}^{p-1} \Delta c d x .
\end{aligned}
$$

Using the Galiardo-Nirenberg-Sobolev inequality for the second term

$$
\int_{\mathbb{R}^{2}} v^{4} d x \leq C \int_{\mathbb{R}^{2}} v^{2} d x \int_{\mathbb{R}^{2}}|\nabla v|^{2} d x
$$

leads to

$$
\begin{aligned}
& \left|\int_{\mathbb{R}^{2}}(n-k)_{+}^{p} \Delta c d x\right| \\
\leq & \left(\int_{\mathbb{R}^{2}}(n-k)_{+}^{2 p} d x\right)^{1 / 2}\|\Delta c\|_{2} \\
\leq & \left(\int_{\mathbb{R}^{2}}(n-k)_{+}^{p} d x\right)^{1 / 2}\left(\int_{\mathbb{R}^{2}}\left|\nabla(n-k)_{+}^{p / 2}\right|^{2} d x\right)^{1 / 2}\|\Delta c\|_{2} \\
\leq & \delta C(p)\|\Delta c\|_{2}^{2} \int_{\mathbb{R}^{2}}(n-k)_{+}^{p} d x+\frac{2}{\delta p} \int_{\mathbb{R}^{2}}\left|\nabla(n-k)_{+}^{p / 2}\right|^{2} d x .
\end{aligned}
$$

Moreover, by interpolation and the same Galiardo-Nirenberg-Sobolev inequality as above, we have for $p \geq 3 / 2$ that

$$
\begin{aligned}
& \left|\int_{\mathbb{R}^{2}}(n-k)_{+}^{p-1} \Delta c d x\right| \\
\leq & \left(\int_{\mathbb{R}^{2}}(n-k)_{+}^{2(p-1)} d x\right)^{1 / 2}\|\Delta c\|_{2} \\
\leq & \left(C(M, p)+\int_{\mathbb{R}^{2}}(n-k)_{+}^{2 p} d x\right)^{1 / 2}\|\Delta c\|_{2} \\
\leq & C(M, p)\|\Delta c\|_{2}+\delta\|\Delta c\|_{2}^{2} \int_{\mathbb{R}^{2}}(n-k)_{+}^{p} d x+\frac{p-1}{\delta p^{2} k} \int_{\mathbb{R}^{2}}\left|\nabla(n-k)_{+}^{p / 2}\right|^{2} d x .
\end{aligned}
$$

Using the estimate

$$
\begin{aligned}
\int_{\mathbb{R}^{2}}(n-k)_{+}^{p+1} d x & =\int_{\mathbb{R}^{2}}\left((n-k)_{+}^{(p+1) / 2}\right)^{2} d x \leq C\left(\int_{\mathbb{R}^{2}}\left|\nabla(n-k)_{+}^{(p+1) / 2}\right| d x\right)^{2} \\
& \leq C(p)\left(\int_{\mathbb{R}^{2}}(n-k)_{+}^{1 / 2}\left|\nabla(n-k)_{+}^{p / 2}\right| d x\right)^{2} \\
& \leq C(p) \int_{\mathbb{R}^{2}}(n-k)_{+} d x \int_{\mathbb{R}^{2}}\left|\nabla(n-k)_{+}^{p / 2}\right|^{2} d x
\end{aligned}
$$


and the fact that $\int_{\mathbb{R}^{2}}(n-k)_{+} d x$ can be made small when choosing $k$ large, we obtain for $p \geq 2$

$$
\begin{aligned}
\frac{d}{d t} \int_{\mathbb{R}^{2}}(n-k)_{+}^{p} d x=( & -1)\left(1-\frac{1}{p C(p) \omega(T, k)}\right) \int_{\mathbb{R}^{2}}(n-k)_{+}^{p+1} d x \\
& +C\left(1+\|\Delta c\|_{2}^{2}\right) \int_{\mathbb{R}^{2}}(n-k)_{+}^{p} d x+C\|\Delta c\|_{2}^{2}+p k^{2} M+C .
\end{aligned}
$$

For fixed $p$, we choose $k=k(p, T)$ sufficiently large such that

$$
1-\frac{1}{p C(p) \omega(T, k)}<0
$$

Using

$$
\begin{aligned}
\int_{\mathbb{R}^{2}}(n-k)_{+}^{p} d x & \leq\left(\int_{\mathbb{R}^{2}}(n-k)_{+} d x\right)^{1 / p}\left(\int_{\mathbb{R}^{2}}(n-k)_{+}^{p+1} d x\right)^{1-1 / p} \\
& \leq\left(\int_{\mathbb{R}^{2}}(n-k)_{+} d x\right)^{1 / p}\left(\int_{\mathbb{R}^{2}}(n-k)_{+}^{p+1} d x\right)^{1-1 / p}
\end{aligned}
$$

we achieve the following differential inequality for $Y_{p}(t), p \geq 2$ and $0<t \leq T$ :

$$
\frac{d}{d t} Y_{p}(t) \leq-(p-1) M^{1 /(p-1)} \delta Y_{p}^{\beta}(t)+C\left(1+\|\Delta c(t)\|_{2}^{2}\right) Y_{p}(t)+C\left(1+\|\Delta c(t)\|_{2}^{2}\right)
$$

with $\beta=\frac{p}{p-1}$.

Second step: Using ODE theory, we obtain that

$$
Y_{p}(t) \leq C(T) \frac{1}{t^{p-1}}
$$

see [10] for details.

Third step: Using $x^{p} \leq 2^{p}(x-k)^{p}$ for $x \geq 2 k$, we observe that

$$
\begin{aligned}
\int_{\mathbb{R}^{2}} n^{p} d x & =\int_{\{n \leq 2 k\}} n^{p} d x+\int_{\{n>2 k\}} n^{p} d x \\
& \leq(2 k)^{p-1} M+2^{p} \int_{\{n>2 k\}}(n-k)^{p} d x \leq(2 k)^{p-1} M+2^{p} \int_{\mathbb{R}^{2}}(n-k)_{+}^{p} d x .
\end{aligned}
$$

Therefore together with (A.10), we obtain (3.14)

$$
\int_{\mathbb{R}^{2}} n^{p} d x \leq C(t)\left(1+t^{\prime 1-p}\right), \quad \forall 0<t^{\prime} \leq t
$$

for $p \geq 2$. For $1<p<2$, the theorem holds by interpolation.

Appendix B. This publication is based on work supported by Award No. KUKI1-007-43, made by King Abdullah University of Science and Technology (KAUST). A. Lorz would like to thank K. Fellner, P. Markowich, T. Pedley, B. Perthame, and M.-T. Wolfram for useful discussions. Moreover, A. Lorz acknowledges support from the ANR Samovar (France). 


\section{REFERENCES}

[1] H.C. Berg, E. coli in Motion, Springer-Verlag, 2003.

[2] P. Biler, Existence and asymptotics of solutions for a parabolic-elliptic system with nonlinear no-flux boundary conditions, Nonli. Anal. T. M. A., 19, 1121-1136, 1992.

[3] P. Biler and T. Nadzieja, Existence and nonexistence of solutions for a model of gravitational interaction of particles, Colloq. Math., 66, 319-334, 1994.

[4] A. Blanchet, J.A. Carrillo, and N. Masmoudi, Infinite time aggregation for the critical PatlakKeller-Segel model in $\mathbb{R}^{2}$, Commun. Pure Appl. Math., 61, 1449-1481, 2008.

[5] A. Blanchet, J. Dolbeault, and B. Perthame, Two-dimensional Keller-Segel model: Optimal critical mass and qualitative properties of the solutions, Electro. J. Diff. Eqs., 44, 32, (electronic) 2006.

[6] M. Burger, M. Di Francesco, and Y. Dolak-Struß, The Keller-Segel model for chemotaxis with prevention of overcrowding: Linear vs. nonlinear diffusion, SIAM J. Math. Anal., 38, 1288-1315, 2006.

[7] H.M. Byrne and M.R. Owen, A new interpretation of the Keller-Segel model based on multiphase modelling, J. Math. Biol., 49(6), 604-626, 2004.

[8] R. Caflisch and G.C. Papanicolaou, Dynamic theory of suspensions with Brownian effects, SIAM J. Appl. Math., 43, 885-906, 1983.

[9] V. Calvez and J.A. Carrillo, Volume effects in the Keller-Segel model: Energy estimates preventing blow-up, J. Math. Pures Appl., 86, 155-175, 2006.

[10] V. Calvez and L. Corrias, The Parabolic-parabolic Keller-Segel Model in $\mathbb{R}^{2}$, Commun. Math. Sci., 6, 417-447, 2008.

[11] J. Carrillo and T. Goudon, Stability and asymptotic analysis of a fluid-particle interaction model, Commun. Part. Diff. Eqs., 31, 1349-1379, 2006.

[12] F. Chalub, Y. Dolak-Struss, P. Markowich, D. Oelz, C. Schmeiser, and A. Soreff, Model hierarchies for cell aggregation by chemotaxis, Math. Models Methods Appl. Sci., 16, 1173-1197, 2006.

[13] L. Corrias and B. Perthame, Critical space for the parabolic-parabolic Keller-Segel model in $\mathbb{R}^{n}$, C.R. Acad. Sci. Paris, Ser. I, 342, 745-750, 2006.

[14] L. Corrias, B. Perthame, and H. Zaag, Global solutions of some chemotaxis and angiogenesis systems in high space dimensions, Milan J. Math., 72, 1-28, 2004.

[15] M. Di Francesco, A. Lorz, and P. Markowich, Chemotaxis-fluid coupled model for swimming bacteria with nonlinear diffusion: Global existence and asymptotic behavior, Discrete Contin. Dyn. Syst. Ser. A, 28(4), 1437-1453, 2010.

[16] R.J. Duan, A. Lorz, and P. Markowich, Global solutions to the coupled chemotaxis-fluid equations, Commun. Part. Diff. Eqs., 35, 1635-1673, 2010.

[17] F. Filbet, A finite volume scheme for the Patlak-Keller-Segel chemotaxis model, Numer. Math., 104, 457-488, 2006.

[18] Y. Giga and H. Sohr, Abstract $L^{p}$ estimates for the Cauchy problem with applications to the Navier-Stokes equations in exterior domains, J. Funct. Anal., 102, 72-94, 1991.

[19] K. Hamdache, Global existence and large time behaviour of solutions for the Vlasov-Stokes equations, Japan J. Indust. Appl. Math., 15, 51-74, 1998.

[20] T. Hillen and K. Painter, Global Existence for a parabolic chemotaxis model with prevention of overcrowding, Adv. in Appl. Math., 26(4), 280-301, 2001.

[21] A.J. Hillesdon, T.J. Pedley, and J.O. Kessler, The development of concentration gradients in a suspension of chemotactic bacteria, Bull. Math. Bio., 57, 299-344, 1995.

[22] D. Horstmann, From 1970 until present: The Keller-Segel model in chemotaxis and its consequences, II, Jahresber. Deutsch. Math.-Verein., 106, 51-69, 2004.

[23] W. Jäger and S. Luckhaus, On explosions of solutions to a system of partial differential equations modelling chemotaxis, Trans. Amer. Math. Soc., 329, 819-824, 1992.

[24] R. Kowalczyk, Preventing blow-up in a chemotaxis model, J. Math. Anal. Appl., 305(2), 566588,2005

[25] O.A. Ladyzhenskaya, Mathematical Theory of Viscous Incompressible Flow, Gordon and Breach Science Publishers Inc., 1968.

[26] P.L. Lions, Mathematical Topics in Fluid Mechanics, Oxford Lecture Series in Mathematics and its Applications, The Clarendon Press Oxford University Press, 1(3), 1996.

[27] J.G. Liu and A. Lorz, A Coupled Chemotaxis-Fluid Model: Global Existence, Ann I. H. Poincaré - AN, 10.1016/j.anihpc.2011.04.005, 2011.

[28] A. Lorz, Coupled chemotaxis fluid model, Math. Models Meth. Appl. Sci., 20, 987-1004, 2010.

[29] A. Marrocco, Numerical simulation of chemotactic bacteria aggregation via mixed finite elements, M2AN Math. Model. Numer. Anal., 37, 617-630, 2003. 
[30] T.J. Pedley and J.O. Kessler, Hydrodynamic phenomena in suspensions of swimming microorganisms, Annu. Rev. Fluid Mech., 24, 313-358, 1992.

[31] B. Perthame, Transport Equation in Biology, Birkhaeuser, 2007.

[32] J.E. Roberts, and J.M. Thomas, Mixed and Hybrid Methods, Handbook of Numerical Analysis, North-Holland, 1993.

[33] I. Tuval, L. Cisneros, C. Dombrowski, C.W. Wolgemuth, J.O. Kessler, and R.E. Goldstein, Bacterial swimming and oxygen transport near contact lines, Proc. Natl. Acad. Sci. USA, 102(7), 2277-2282, 2005.

[34] M. Winkler, Global large-data solutions in a chemotaxis-(Navier-)Stokes system modeling cellular swimming in fluid drops, Commun. Part. Diff. Eqs., to appear, 2011.

[35] www.freefem.org. 